\title{
Morbilidad ambulatoria y hospitalaria en el Perú. Cambios entre los años 2011 y 2015
}

\author{
Ambulatory and hospital morbidity in Peru. Changes between the years 2011 and 2015
}

\section{Sr. Editor:}

La mayor parte de la atención médica en el Perú se realiza en la consulta ambulatoria y la atención en hospitalización. Por ello, es importante conocer las patologías que manejamos todos los médicos y las instituciones de salud del país, no solo para dirigir mejor todos los recursos de cada institución, sino para dirigir mejor la actualización de los conocimientos de los médicos que ya atienden pacientes y también para planificar mejor la enseñanza de la medicina en las universidades.

Aunque el análisis de la estructura de la morbilidad en el Perú es realizado año a año por el Ministerio de Salud (MINSA), y dichos resultados son publicados en sendos documentos mediante estadísticas oficiales en su página web (1), estos datos pueden ser de difícil disponibilidad para la mayoría de los médicos. Por ello, revisamos las estadísticas publicadas en la página web oficial del MINSA del año 2011 al $2015(1,2)$ y las resumimos en dos tablas, una sobre la patología ambulatoria y otra sobre la morbilidad hospitalaria.

En cuanto a la morbilidad de consulta ambulatoria (tabla 1), en el Perú en el año 2015 se atendieron casi 35 millones consultas médicas ambulatorias, lo cual fue un aumento de 36,3\% respecto al año 2011. Las patologías médicas más frecuentes en consulta ambulatoria en el Perú en el 2015 (tabla 1), fueron las infecciones agudas de vías aéreas superiores (IRA) con $16,7 \%$ del total y las infecciones intestinales $(5,4 \%)$. En tercer y cuarto lugar estaban las enfermedades del esófago, estómago y duodeno y las del sistema urinario, con 3.2\% cada una, respectivamente, y luego, las dorsopatías $(3,2 \%)$ y la obesidad $(3,0 \%)$.

Si comparamos estos resultados con la atención ambulatoria en Estados Unidos (3), en el año 2015, las cuatro patologías ambulatorias más frecuentes fueron enfermedades crónicas como artropatías, hipertensión arterial, desórdenes espinales y diabetes mellitus, dejando en quinto lugar a las infecciones agudas respiratorias altas. En otros países de Sudamérica (4) se observaron resultados más similares a Perú: las consultas ambulatorias más frecuentes fueron las infecciones respiratorias altas agudas, llegando hasta casi $50 \%$ del total de atenciones en atención primaria en Ecuador.

Con respecto a la morbilidad de hospitalización (tabla 2), en el Perú en el 2015 hubo casi 900 mil atenciones en hospitalización, que hace una relación de 2,58 atenciones hospitalarias por cada 100 atenciones en consulta ambulatoria en dicho año. Sin contar las hospitalizaciones por gineco-obstetricia (abortos y atenciones del embarazo) y quirúrgicas (cirugías de apéndice y vesícula), las cinco patologías médicas más frecuentes en hospitalización fueron tumores malignos $(2,7 \%)$, influenza y neumonía $(2,3 \%)$, enfermedades infecciosas intestinales $(1,6 \%)$, otras enfermedades del sistema urinario $(1,3 \%)$ y las infecciones de la piel y tejido celular subcutáneo $(1,1 \%)$.

Estos resultados fueron similares en Estados Unidos en el año 2010, donde las tres patologías médicas más frecuentes de hospitalización fueron las enfermedades cardíacas, neoplasias malignas y

\footnotetext{
Hospital Nacional Arzobispo Loayza.

Facultad de Medicina. Universidad Peruana Cayetano Heredia.

Médico Internista.

Profesor Principal

Profesor Auxiliar.
} 
Tabla 1. Principales diagnósticos de atención en consulta ambulatoria en el Perú en los años 2011 y 2015 (se incluye diagnósticos de ginecología y odontología).

\begin{tabular}{|c|c|c|c|c|c|c|}
\hline \multirow{2}{*}{ ORD } & \multirow{2}{*}{ CAUSAS DE MORBILIDAD } & \multicolumn{2}{|c|}{2011} & \multicolumn{2}{|c|}{2015} & \multirow{2}{*}{$\begin{array}{c}\% \\
\text { variación }\end{array}$} \\
\hline & & $\mathbf{n}$ & $\%$ & $\mathbf{n}$ & $\%$ & \\
\hline & TOTAL & 25598494 & 100,0 & 34882652 & 100,0 & 36,3 \\
\hline 1 & INFECCIONES AGUDAS DE LAS VIAS RESPIRATORIAS SUPERIORES (J00 - J06) & 5771689 & 22,5 & 5813789 & 16,7 & 0,7 \\
\hline 2 & $\begin{array}{l}\text { ENFERMEDADES DE LA CAVIDAD BUCAL, DE LAS GLANDULAS SALIVALES Y DELOS } \\
\text { MAXILARES (K00 - K14) }\end{array}$ & 2708081 & 10,6 & 5295343 & 15,2 & 95,5 \\
\hline 3 & ENFERMEDADES INFECCIOSAS INTESTINALES (A00 - A09) & 1345651 & 5,3 & 1177095 & 3,4 & $-12,5$ \\
\hline 4 & OTRAS ENFERMEDADES DEI SISTEMA URINARIO (N30 - N39) & 915303 & 3,6 & 1111200 & 3,2 & 21,4 \\
\hline 5 & DESNUTRICION (E40 - E46) & 873271 & 3,4 & 884352 & 2,5 & 1,3 \\
\hline 6 & ENFERMEDADES DEL ESOFAGO, DEL ESTOMAGO Y DEL DUODENO (K20 - K31) & 798663 & 3,1 & 1112143 & 3,0 & 39,3 \\
\hline 7 & DORSOPATIAS (M40 - M54) & 791585 & 3,1 & 1106368 & 3,2 & 39,8 \\
\hline 8 & HELMINTIASIS (B65 - B83) & 761653 & 3,0 & 549869 & 1,6 & $-27,8$ \\
\hline 9 & ENFERMEDADES CRONICAS DE LAS VIAS RESPIRATORIAS INFERIORES (J40 - J47) & 649456 & 2,5 & 601071 & 1,7 & $-7,5$ \\
\hline 10 & $\begin{array}{l}\text { OTROS TRASTORNOS MATERNOS RELACIONADOS PRINCIPALMENTE CONEI } \\
\text { EMBARAZO (O20 - O29) }\end{array}$ & 561096 & 2,2 & 812891 & 2,3 & 44,9 \\
\hline 11 & OTRAS INFECCIONES AGUDAS DE LAS VIAS RESPIRATORIAS INFERIORES (J20 - J22) & 535299 & 2,1 & 391312 & 1,1 & $-26,9$ \\
\hline 12 & INFECCIONES C/MODO DE TRANSMISION PREDOMINANTEMENTE SEXUAL (A50 - A64) & 489098 & 1,9 & 699158 & 2,0 & 42,9 \\
\hline 13 & DERMATITIS Y ECZEMA (L20 - L30) & 440953 & 1,7 & 456200 & 1,3 & 3,5 \\
\hline 14 & MICOSIS (B35 - B49) & 423151 & 1,7 & 449789 & 1,3 & 6,3 \\
\hline 15 & OBESIDAD Y OTROS DE HIPERALIMENTACION (E65 - E68) & 398707 & 1,6 & 1059149 & 3,0 & 165,6 \\
\hline 16 & TRASTORNOS EPISODICOS Y PAROXISTICOS (G40 - G47) & 343982 & 1,3 & 551961 & 1,0 & 60,5 \\
\hline 17 & ARTROPATIAS (M00 - M25) & 336470 & 1,3 & 605461 & 1,7 & 79,9 \\
\hline 18 & ANEMIAS NUTRICIONALES (D50 - D53) & 317649 & 1,2 & 736833 & 2,1 & 132,0 \\
\hline \multirow[t]{3}{*}{19} & ENFERMEDADES HIPERTENSIVAS (110 - 115) & & & 374027 & 1,1 & No aplicable \\
\hline & $\begin{array}{l}\text { SINTOMAS, SIGNOS Y HALLAZGOS ANORMALES CLINICOS Y DE LABORATORIO, NO } \\
\text { CLASIFICADOS EN OTRA PARTE (R00-R99) }\end{array}$ & 1421231 & 5,6 & 1820099 & 5,2 & \\
\hline & LAS DEMAS CAUSAS & 7136737 & 27,9 & 9274542 & 26,6 & \\
\hline
\end{tabular}

Fuente: http://w w w .minsa.gob.pe/estadisticas/estadisticas/morbilidad/cemacros.asp?00

neumonía (5) y en Ecuador, donde en el año 2014 las tres patologías más freuentes en hospitalización fueron la diarrea infecciosa, neumonía y otros trastornos del sistema urinario (6).

Al comparar los diagnósticos en la atención ambulatoria entre los años 2011 y 2015, en la población general del Perú se observó un incremento de más de 2,5 veces el número de atenciones ambulatorias por obesidad, siendo la séptima causa de atención ambulatoria en el Perú en el año 2015. Esto tendría relación con los profundos cambios en el estilo de vida que existen en nuestra sociedad en los últimos años, sobre todo por malos hábitos de alimentación, como el aumento del consumo de alimentos ultraprocesados generados por la industria alimentaria, y el aumento del sedentarismo. Esto ha conllevado a un incremento de la pandemia de la obesidad a nivel global, y en el Perú esto se evidencia sobre todo en adultos menores de 30 años y niños mayores de 5 años y adolescentes (7).

Por otro lado, en el mismo periodo, también se observó un aumento de 2,5 veces en las atenciones por anemias nutricionales. Esto concuerda con los reportes del MINSA sobre la alta prevalencia $(43,6 \%)$ de anemia en niños y niñas de 6 a 36 meses de edad, lo cual llevó a la publicación en abril del año 2017 de un plan nacional para el control de la anemia maternoinfantil (8).

Por último, al comparar los diagnósticos en hospitalización entre los años 2011 y 2015, se observó una disminución de casi el $70 \%$ en el número de hospitalizaciones por complicaciones del parto, lo cual probablemente sea por un aumento de la tasa de cesáreas en ese lapso, ya que se reportó hasta 50\% de tasa de cesárea en el año 2017 en un hospital 
Tabla 2. Principales diagnósticos de hospitalización en el Perú entre los años 2011 y 2015.

\begin{tabular}{|c|c|c|c|c|c|c|}
\hline \multirow{2}{*}{ ORDEN } & \multirow{2}{*}{ CAUSA DE MORBILIDAD EN HOSPITALIZACIÓN } & \multicolumn{2}{|c|}{2011} & \multicolumn{2}{|c|}{2015} & \multirow{2}{*}{$\begin{array}{c}\% \\
\text { variación }\end{array}$} \\
\hline & & CASOS & $\%$ & CASOS & $\%$ & \\
\hline & TOTAL & 828843 & 100,0 & 899280 & 100,0 & 8,5 \\
\hline 1 & Embarazo terminado en aborto $(\mathrm{O} 00-\mathrm{O} 08)$ & 40819 & 4,9 & 46157 & 5,1 & 13,1 \\
\hline 2 & Enfermedades del apéndice (K35-K38) & 28610 & 3,5 & 32928 & 3,7 & 15,1 \\
\hline 3 & Trastornos de la vesícula biliar, de las vías biliares y del páncreas (K80-K87) & 27479 & 3,3 & 32451 & 3,6 & 18,1 \\
\hline 4 & $\begin{array}{l}\text { Atención materna relacionada con el feto y la cavidad amniótica y con posibles problemas } \\
\text { del parto (O30-O48) }\end{array}$ & 25511 & 3,1 & 12173 & 1,4 & $-52,3$ \\
\hline 5 & Influenza [gripe] y neumonía (J09-J18) & 24055 & 2,9 & 20473 & 2,3 & $-14,9$ \\
\hline 6 & Otros trastornos maternos relacionados principalmente con el embarazo (O20-O29) & 22282 & 2,7 & 26681 & 3,0 & 19,7 \\
\hline 7 & Complicaciones del trabajo de parto y del parto (O60-O75) & 20219 & 2,4 & 6294 & 0,7 & $-68,9$ \\
\hline 8 & Enfermedades infecciosas intestinales (A00-A09) & 15423 & 1,9 & 14477 & 1,6 & $-6,1$ \\
\hline 9 & Traumatismos de la cabeza (S00-S09) & 12795 & 1,5 & 9876 & 1,1 & $-22,8$ \\
\hline 10 & Infecciones especificas del periodo perinatal (P35-P39) & 12084 & 1,5 & 14081 & 1,6 & 16,5 \\
\hline 11 & Trastornos hemorrágicos y hematológicos del feto y del recién nacido (P50-P61) & 10829 & 1,3 & 11880 & 1,3 & 9,7 \\
\hline 12 & Hernia (K40-K46) & 10704 & 1,3 & 13843 & 1,5 & 29,3 \\
\hline 13 & Trastornos relacionados con la duración de la gestación y el crecimiento fetal (P05-P08) & 10704 & 1,3 & 10676 & 1,2 & $-0,3$ \\
\hline 14 & Trastornos no inflamatorios de los órganos genitales femeninos (N80-N98) & 10678 & 1,3 & 13274 & 1,5 & 24,3 \\
\hline 15 & Infecciones de la piel y del tejido subcutáneo (L00-L08) & 9244 & 1,1 & 9926 & 1,1 & 7,4 \\
\hline 16 & Enfermedades crónicas de las vías respiratorias inferiores (J40-J47) & 9200 & 1,1 & 10346 & 1,2 & 12,5 \\
\hline 17 & Otras enfermedades del sistema urinario (N30-N39) & 8873 & 1,1 & 12102 & 1,3 & 36,4 \\
\hline 18 & Enfermedades cerebrovasculares (160-169) & 7021 & 0,8 & 7026 & 0,8 & 0,1 \\
\hline 19 & Trastornos respiratorios y cardiovasculares especificos del periodo perinatal (P20-P29) & 6864 & 0,8 & 7531 & 0,8 & 9,7 \\
\hline 20 & Diabetes mellitus (E10-E14) & 6853 & 0,8 & 8008 & 0,9 & 16,9 \\
\hline 21 & Otras infecciones agudas de las vías respiratorias inferiores (J20-J22) & 4605 & 0,6 & 6030 & 0,7 & 30,9 \\
\hline \multirow[t]{2}{*}{22} & TUMORES (NEOPLASIAS) MALIGNOS (C00 - C97) & \multicolumn{2}{|l|}{ No registrado } & 24466 & 2,7 & No aplicable \\
\hline & Las demás causas & 503991 & 61,0 & 548581 & 61,0 & 8,9 \\
\hline
\end{tabular}

Fuente: http://w w w .minsa.gob.pe/estadisticas/estadisticas/morbilidad/hsmacros.asp?00

público de Lima-Perú (9). Por otro lado, se observó una disminución de hospitalizaciones por influenza y neumonía, probablemente por el mayor acceso y disponibilidad a las vacunas tanto de influenza como neumococo como lo ha planificado el MINSA en los últimos años (10).

\section{Otto Barnaby Guillén López 1,a; 2,c; Jorge Alberto Casas Castañeda ${ }^{1, a ; 2, b}$}

\section{Correspondencia:}

Otto Barnaby Guillén-López

Avenida Alfonso Ugarte 848, Lima - 01, Perú

Correo electrónico: otto.guillen.1@upch.pe

\section{REFERENCIAS BIBLIOGRÁFICAS}

1. Ministerio de Salud del Perú. Principales causas de morbilidad en consulta externa de establecimientos
MINSA y gobiernos regionales Perú - Año 2015. (citado el 27 de febrero de 2016). Disponible en: www.minsa.gob.pe/estadisticas/estadisticas/ Morbilidad/CEMacros.asp?00

2. Ministerio de Salud, Perú. PRINCIPALES CAUSAS DE MORBILIDAD DE HOSPITALIZACION en el PERU. (citado el 27 de junio de 2017). Disponible en: http://www.minsa.gob.pe/estadisticas/ estadisticas/morbilidad/hsmacros.asp?00

3. National Ambulatory Medical Care Survey: 2015 State and National Summary Tables. (citado el 21 de julio de 2018). Disponible en: https://www.cdc.gov/ nchs/data/ahcd/namcs_summary/2015_namcs_web_ tables.pdf

4. Ministerio de Salud de Chile (MINSAL). Consultas médicas realizadas en atención primaria, por Región y Servicio de salud, SNSS 2014. (citado el 21 de julio de 2018). Disponible en: https://reportesdeis.minsal. c 1/ R E M / 2014 / R E M 04 S E C C I O A / REM04SECCIONA.aspx 
5. Centers for Disease Control and Prevention. Number and rate of discharges from short-stay hospitals and of days of care, with average length of stay and standard error, by selected first-listed diagnostic categories: United States, 2010. (citado el 21 de julio de 2018). Disponible en: https://www.cdc.gov/nchs/ data/nhds/2average/2010ave2_firstlist.pdf

6. Ministerio de salud pública del Ecuador. Principales causas de morbilidad hospitalaria-general. (citado el 23 de julio de 2018). Disponible en: https://public. tableau.com/profile/javier.gaona\#!/vizhome/egresosh ospitalariosinec2014/1rascausasdemorbilidad

7. Pajuelo Ramírez J. La obesidad en el Perú. An Fac med. 2017; 78(2):179-185. DOI: http://dx.doi. org/10.15381/anales.v78i2.13214.
8. Ministerio Salud, Perú. Plan Nacional para la Reducción y control de la anemia materno-infantil y la desnutrición crónica infantil en el Perú: 2017-2021. (citado el 23 de julio de 2018). Disponible en: ftp:// ftp2.minsa.gob.pe/normaslegales/2017/RM_2492017-MINSA.PDF

9. Barrena M, Quispesaravia P. Frecuencia e indicaciones del parto por cesárea en un hospital público de Lima en el período enero 2013 - diciembre 2017. Tesis de bachiller en medicina. Lima, Perú: Universidad Peruana Cayetano Heredia; 2018. 31 pp.

10. MINSA - Perú. Documento Técnico: Plan de Trabajo Semana de Vacunación en las Américas. Perú 2014. (citado el 07 de marzo de 2019). Disponible en: https://www.paho.org/vwa/wp-content/ uploads/2014/03/Peru.pdf

Recibido: 11/03/2019 